\title{
Associations between cognitive impairment and cerebrovascular reserve in different lobes during the acute stroke of anterior circulations
}

\section{Mengna Chu}

The Third Affiliated Hospital of Anhui Medical University (The First people's Hospital of Hefei)

\section{Wei Dong}

the First Affiliated Hospital of Anhui University of Chinese Medicine

Chao Huang

The Third Affiliated Hospital of Anhui Medical University (The First people's Hospital of Hefei)

Bin Dong ( $\nabla$ dongbin676@163.com)

The Third Affiliated Hospital of Anhui Medical University (The First people's Hospital of Hefei)

\section{Ming Yao}

The Fourth Affiliated Hospital of Anhui Medical University

\section{Research Article}

Keywords: Cognitive impairment, cerebrovascular reserve, acute stroke, anterior circulations

Posted Date: January 28th, 2022

DOl: https://doi.org/10.21203/rs.3.rs-1139963/v1

License: (9) This work is licensed under a Creative Commons Attribution 4.0 International License. Read Full License 


\section{Abstract \\ Background}

Cognitive impairments at the acute stroke phase should not be ignored, and its potential risk factors and mechanism need to be clinically assessed. Recently, various studies revealed that reduced cerebrovascular reserve (CVR) predicts cognitive declines such as mild cognitive impairment (MCl) and dementia. However, the association of CVR in different lobes with cognitive impairment at the acute stroke phase remains unclear. In this study, we analyzed the correlation between CVR in different lobes and cognitive impairment at the acute stroke phase in cerebral infarction patients.

\section{Method}

This study included 125 subjects: 96 in the acute stroke phase (38 left infarction and 58 right infarction) and 29 healthy controls with no history of neurological disease. The basic sociodemographic characteristics of the two groups matched and their cognitive status was assessed by the Montreal Cognitive Assessment (MoCA). The CVR was assessed by CT perfusion imaging scans, including cerebral blood flow (CBF), cerebral blood volume (CBV), time to peak (TTP), and mean transit time (MTT).

\section{Results}

The MoCA scores of visuospatial/executive, attention, and orientation in both left and right infarctions patients significantly decreased. Nevertheless, the MoCA scores for naming, language, and delayed recall significantly only decreased in left infarction patients. The CBF of right vessels in the frontal lobe and the MTT of left vessels in the occipital lobe was negatively related to the MoCA scores of left infarction patients. The CBV of left vessels in the frontal lobe and CBF of left vessels in the parietal lobe were positively related to the MoCA scores of left infarction patients. The CBF of right vessels in the temporal lobe was negatively related to the MoCA scores of right infarction patients. Finally, the CBF of the left vessels in the temporal lobe was positively related to the MoCA scores of right infarction patients.

\section{Conclusions}

CVR was closely related to cognitive impairment at the acute stroke phase. Decreasing CVR might be a potential neuroimaging biomarker to predict cognitive impairment at the acute stroke phase.

\section{Introduction}

It has been demonstrated that cerebral infarction often causes cognitive dysfunction, also named poststroke cognitive impairment (PSCl), ranging from mild cognitive impairment ( $\mathrm{MCl}$ ) to dementia. Approximately $23-30 \%$ of cerebral infarction patients develop $\mathrm{MCl}$ or dementia up to 4 years after 
strokes $^{1,2}$. The 2016 American Heart Association/American Stroke Association (AHA/ASA) Guidelines recommended that the cognitive status of stroke patients should be screened during hospitalization in the acute stroke phase. The early detection of cognitive impairment in the acute stroke phase is the first step to reducing vascular risk factors and improving outcomes ${ }^{3,4}$. Therefore, cognitive impairments at the acute stroke phase should not be ignored, and its potential risk factors and mechanism need to be clinically assessed.

Moreover, CVR reflects the capacity of the brain to adequately maintain blood flow when perfusion pressure decreases. Recently, various studies revealed that reduced CVR can also predict cognitive declines such as $\mathrm{MCl}$ and dementia ${ }^{5,6}$. CVR might be a potential biomarker to monitor pressure-perfusioncognition relationships ${ }^{7}$. However, at present, the association of CVR with cognitive impairments at the acute stroke phase remains unclear. Therefore, in the present study, we analyzed the correlation between CVR in different lobes and cognitive impairments at the acute stroke phase in cerebral infarction patients.

\section{Methods}

\section{Subjects}

We included 249 patients with new-onset cerebral infarction hospitalized at the Neurology Department in the Third Affiliated Hospital of Anhui Medical University between August 2019 and July 2021. The first onset time of these patients was $<72 \mathrm{~h}$, and they were diagnosed by diffusion-weighted imaging (DWI). Patients with posterior circulation cerebral infarction, cognitive impairment before the stroke, serious liver history of kidney and heart diseases, surgical history, allergy to iodine, severe aphasia, and inability to cooperate with the scale were excluded (Figure 1). Healthy controls without cerebral infarction were also analyzed. The Ethics Committee of The Third Affiliated Hospital of Anhui Medical University (The First people's Hospital of Hefei) approved this study [No. 2016(51)]. Informed consent was obtained from all participants involved in the study. All study procedures were conducted in accordance with the Declaration of Helsinki.

\section{Assessment of cognitive functions}

The Montreal Cognitive Assessment (MoCA) can be used as a brief cognitive screening strategy in acute stroke settings ${ }^{4,8}$. Previously, the assessment of cognitive functions by $\mathrm{MoCA}^{9}$ was performed during hospital treatment in the acute phase of ischemic stroke (first four weeks after stroke onset) ${ }^{10}$. Seven cognitive scores were calculated: language, naming, attention, abstraction, delayed recall, visuospatial function, and orientation ${ }^{9}$. According to the recommended cutoffs, cognitive functions were categorized as follows: 0 to 19 (severe cognitive impairment), 20 to 24 (mild cognitive impairment), and 25 to 30 (no cognitive impairment) ${ }^{11}$.

\section{CVR assessment by CT perfusion imaging scanning}


CT perfusion imaging scanning was performed after a conventional head CT plain scan by a dual-source CT scanner (Somatom Definition Flash, Siemens Healthcare, Forchheim, Germany). The scanning parameters were: tube voltage, $80 \mathrm{kV}$; tube current, $180 \mathrm{~mA}$; slice thickness, $1.5 \mathrm{~mm}$; pitch, $1.0 \mathrm{~mm}$; collimation, $64 \times 0.6 \mathrm{~mm}$; matrix, $512 \times 512$. The scanning was repeated 30 times with $1.5 \mathrm{~s}$ scan time and $4 \mathrm{~s}$ intervals. lohexol $350 \mathrm{mg} / \mathrm{mL}(50 \mathrm{~mL})+$ saline $(40 \mathrm{~mL})$ was intravenously injected into the right median cubital vein at $6 \mathrm{~mL} / \mathrm{s}$ using a double tube high-pressure syringe.

To reduce the vascular structure salience, a computer was used to automatically eliminate vascular pixels. Quantitative evaluation of the CVR was performed using cerebral blood volume (CBV), cerebral blood flow (CBF), mean transit time (MTT), and time to peak (TTP).

\section{Statistical analyses}

The SPSS package (V.13.0) was used for data analyses with a 0.05 statistically significant level. Baseline characteristics, such as education, cardiac function, serum triglyceride level, hypertension, diabetes, smoking status, drinking status, carotid atherosclerosis, atrial fibrillation, serum total cholesterol level, serum high-density lipoprotein level, serum low-density lipoprotein level, homocysteine level, and uric acid level were analyzed. Classified data were represented as $n(\%)$ and continuous data as means \pm SDs. A $\chi^{2}$ test was performed on the data that met the parameter test criteria. Results were analyzed for statistical significance using t-test or Analysis of Variance (ANOVA) followed by Duncan's multiple range tests. The association between CVR and CTP was evaluated by linear regression analyses.

\section{Results}

\section{Baseline characteristics}

At baseline, 96 patients with new-onset anterior circulation cerebral infarction were included in the observational analyses. Their mean age was 63.93 [standard deviation (SD) 10.05] and 67 participants (69.79\%) were men. According to the lesion location, patients were divided into left and right infarction groups. The baseline characteristics of participants (96 patients and 29 controls) are shown in Table 1. No significant differences in baseline characteristics were detected among participants, except for homocysteine levels.

\section{Cognitive impairment at the acute stroke phase}

Cognitive impairment (MoCA score < 25) appeared more in patients with new-onset anterior circulation cerebral infarction than in controls ( 56.3 versus $27.6 \% ; p=0.007$ ). As shown in Figure $2 \mathrm{~A}$, the proportion of mild cognitive impairment (MoCA score: 20 to 24 ) in patients was similar to controls (20.8 versus 20.7\%). However, the proportion of severe cognitive impairment (MoCA score: 0 to 19) was higher (35.4 versus $6.9 \%$ ). Additionally, the cognitive impairment of the left infarction group did not differ from the right infarction group (Figure 2B). 
Moreover, no significant differences in the abstraction MoCA score were detected among participants (Table 2). Compared to controls, the visuospatial/executive, attention, and orientation MoCA scores in both left and right infarction patients significantly decreased. Nevertheless, the MoCA scores for naming, language, and delayed recall significantly decreased only in left infarction patients.

\section{Cerebrovascular reserve}

The CBF, CBV, MTT, and TTP in different brain areas, including frontal, temporal, parietal, and occipital lobes, the basal ganglia, and the semioval center were used to assess the CVR of patients with new-onset anterior circulation cerebral infarction (Table 3). In the frontal lobe of patients, only MTT significantly increased compared to controls, and the other CVR predictors such as CBF, CBV, and TTP did not differ.

In the temporal lobe of patients, the CBF, CBV, and MTT significantly differed compared to controls. Interestingly, significant differences for CBF and MTT were detected only in left infarction patients. In these patients, the left vessels CBF significantly decreased and the MTT significantly increased. However, the CBF and MTT of right infarction patients were similar to controls. The right vessels CBV significantly increased in right infarction patients but, for left infarction patients, it was similar to controls.

Regarding the parietal lobe of patients, significant differences were detected for CBF and MTT compared to controls. For right infarction patients, the $\mathrm{CBF}$ in both left and right vessels significantly decreased compared to controls. The left vessels CBF significantly decreased and the MTT significantly increased in left infarction patients. MTT of right vessels also significantly increased in right infarction patients.

In the occipital lobe of patients, significant differences regarding CBV and MTT were detected compared to controls. The left vessels MTT significantly increased in left infarction patients, similar to the right vessels of right infarction patients. Additionally, compared to controls, the right vessels CBV significantly increased in right infarction patients but did not differ in left infarction patients.

Moreover, in the semioval center of patients, there were significant differences in CBF and MTT compared to controls. The left vessels CBF significantly decreased in left infarction patients. A significant increase was also detected for the MTT right vessels of right infarction patients. Similarly, the left vessels MTT significantly increased in both right and left infarction patients.

Finally, in the basal ganglia, the left vessels CBF significantly decreased in left infarction patients. On the other hand, the left vessels MTT significantly increased in the left infarction patients, similar to the right vessels of right infarction patients. Also, the left vessels TTP significantly decreased in right infarction patients.

\section{Associations between cognitive impairment and cerebrovascular reserve}

The data from left infarction patients were selected during linear regression analyses to assess the relationship between cognitive impairment and cerebrovascular reserve. This analysis indicated that the 
right vessels $\mathrm{CBF}$ and left vessels $\mathrm{CBV}$ in the frontal lobe, the left vessels $\mathrm{CBF}$ in the parietal lobe, the left vessels MTT in the occipital lobe were risk factors and composed the regression equation $(p<0.0001$, Table 4). Especially, the right vessels CBF in the frontal lobe and the left vessels MTT in the occipital lobe were negatively related to the MoCA scores of left infarction patients. On the other hand, the left vessels $\mathrm{CBV}$ in the frontal lobe and the left vessels CBF in the parietal lobe were positively related to the MoCA scores of left infarction patients.

The data from right infarction patients was also used in a linear regression analysis to evaluate the relationship between cognitive impairment and cerebrovascular reserve. The results indicated that the $\mathrm{CBF}$ in the temporal lobe was a risk factor, included in the regression equation ( $p=0.010$, Table 4$)$. Especially, the right vessels $\mathrm{CBF}$ in the temporal lobe was negatively related to the MoCA scores of right infarction patients. Besides, the left vessels CBF in the temporal lobe was positively related to the MoCA scores of these patients.

\section{Discussion}

Cognitive function in the acute stroke setting was previously reported to be a predictive factor for PSCI development ${ }^{4,12}$. Lee et al (2020) reported that $63.8 \%$ of patients presented cognitive impairments from acute ischemic strokes (28.8\% with mild cognitive impairment and $35.0 \%$ with severe cognitive impairment $)^{13}$. Hence, patients in the acute stroke phase can present a general decline in cognitive efficiency. In the present study, we found that the proportion of cognitive impairments at the acute stroke phase was $56.3 \%$ (20.8\% mild cognitive impairment and $35.4 \%$ severe cognitive impairment) in patients with new-onset anterior circulation cerebral infarction, consistent with other studies.

The lesion location is also an important determinant for $\mathrm{PSCl}^{14}$. A large cohort of 410 acute ischemic stroke patients revealed that the left anterior circulation, such as angular gyrus and basal ganglia are the strategic structures for $\mathrm{PSCl}^{15}$. Addirionally, Weaver et al (2021) analyzed the data of 2950 acute ischemic stroke patients and concluded that the appearance of infarction in the left frontal lobe, left temporal lobe, left thalamus and right parietal lobe was closely related to $\mathrm{PSCl}^{16}$. Ni et al (2021) proposed that the hemodynamics asymmetry makes the left hemisphere more vulnerable to various risk factors compared to the right hemisphere ${ }^{17}$. In our study, 63.1 and $51.7 \%$ of patients with left and right anterior circulation cerebral infarction presented cognitive impairment, respectively. The proportion of cognitive impairment in the left infarction group was only slightly higher than that in the right infarction one. This might be related to our limited sample size and, as the number of samples increases, this difference might become more prominent.

The CVR reflects the capacity of the brain to adequately maintain blood flow when perfusion pressure decreases. MTT is the most appropriate prediction parameter to evaluate CVR declines ${ }^{18}$. Our study showed that the MTT value in all brain areas (frontal, temporal, parietal, and occipital lobes, basal ganglia, and semioval center) of patients significantly increased compared to controls. Also, the MTT increase on the ipsilateral side of the lesion was more significant. Chen et al (2006) reported that MTT 
increases were more likely to appear in the areas with severely reduced perfusion reserves than in those with moderately reduced or normal perfusion reserves ${ }^{19} . \mathrm{CBF}$ is another index to evaluate $\mathrm{CVR}^{20}$. We detected a significant decrease of CBF in the temporal and parietal lobes, semioval center, and basal ganglia of patients. The CBF decreased more significantly on the ipsilateral side of the lesion. Besides, we showed that the CBV in the right occipital lobe of right cerebral infarction patients significantly increased. Ogasawara et al (2002) considered that the increase of CBV can be an initial indicator of cerebral perfusion pressure and CVR decreases ${ }^{21}$. Altogether, these results demonstrated that the CVR decreased in the patients with new-onset anterior circulation cerebral infarction and this decrease was associated with the lesion. In the present study, we also found that the MoCA scores of left infarction patients were negatively related to the CBF in the right frontal lobe and MTT in the left occipital lobe, while they were positively related to the $\mathrm{CBV}$ in the left frontal lobe and $\mathrm{CBF}$ in the left parietal lobe. Moreover, MoCA scores of right infarction patients were negatively related to $\mathrm{CBF}$ in the right temporal lobe, while positively related to $\mathrm{CBF}$ in the left temporal lobe. These results indicated that regional CBF might be a more sensitive marker of cognitive impairment, consistent with other studies. Yin et al (2019) showed that $\mathrm{CBF}$ could be used as a candidate imaging indicator to monitor global cognitive function changes in patients with cerebral autosomal dominant arteriopathy with subcortical infarcts and leukoencephalopathy ${ }^{22}$.

\section{Conclusions}

Overall, cognitive impairment was associated with CVR in patients with new-onset anterior circulation cerebral infarction, especially at the acute stroke phase. Finally, decreasing CVR might be a potential neuroimaging biomarker to predict the cognitive impairment at the acute stroke phase.

\section{Declarations}

\section{Ethics approval and consent to participate}

The study was conducted according to the guidelines of the Declaration of Helsinki and approved by the the Ethics Committee of The Third Affiliated Hospital of Anhui Medical University (The First people's Hospital of Hefei) [No. 2016(51)].

Informed consent was obtained from all participants involved in the study.

\section{Consent for publication}

Not applicable.

\section{Availability of data and materials}

All data generated or analysed during this study are included in this published article; the original data in this study are available from the corresponding author upon reasonable request. 


\section{Competing interest}

The authors declare that they have no competing interests.

\section{Funding}

This work was supported by Applied Medical Research Project of Hefei Municipal Health and Family Planning Commission in 2017 (hwk2017ZD007) and Scientific Research Fund of Anhui Medical University in 2020(2020xkj237).

\section{Authors' contributions}

B.D. and M.Y. contributed to the concept and design of the study, data acquisition and interpretation. MN.C. and W.D. contributed to data analysis, interpretation, and writing of the manuscript. C.H. revised the manuscript. All authors read and approved the final manuscript.

\section{Acknowledgements}

We thank the participants for agreeing to share their clinical information for this study.

\section{Authors' information}

${ }^{1}$ Department of Neurology, The Third Affiliated Hospital of Anhui Medical University(The First people's Hospital of Hefei), Hefei, 230061, China. ${ }^{2}$ Department of Neurology, the First Affiliated Hospital of Anhui University of Chinese Medicine, Hefei, 230031, China. 3 Department of Neurology, The Fourth Affiliated Hospital of Anhui Medical University, Hefei, 230032, China.

\section{References}

1. Makin SD, Turpin S, Dennis MS, Wardlaw JM. Cognitive impairment after lacunar stroke: systematic review and meta-analysis of incidence, prevalence and comparison with other stroke subtypes. Journal of neurology, neurosurgery, and psychiatry. 2013;84(8):893-900. doi: 10.1136/jnnp-2012303645.

2. Strong $\mathrm{K}$, Mathers $\mathrm{C}$, Bonita R. Preventing stroke: saving lives around the world. The Lancet Neurology. 2007;6(2):182-7. https://doi: 10.1016/S1474-4422(07)70031-5.

3. Godefroy O, Fickl A, Roussel M, Auribault C, Bugnicourt JM, Lamy C, et al. Is the Montreal Cognitive Assessment superior to the Mini-Mental State Examination to detect poststroke cognitive impairment? A study with neuropsychological evaluation. Stroke. 2011;42(6):1712-6. doi: 10.1161/STROKEAHA.110.606277.

4. Horstmann S, Rizos T, Rauch G, Arden C, Veltkamp R. Feasibility of the Montreal Cognitive Assessment in acute stroke patients. European journal of neurology. 2014;21(11):1387-93. doi: 10.1111/ene.12505. 
5. Silvestrini M, Pasqualetti P, Baruffaldi R, Bartolini M, Handouk Y, Matteis M, et al. Cerebrovascular reactivity and cognitive decline in patients with Alzheimer disease. Stroke. 2006;37(4):1010-5. doi: 10.1161/01.STR.0000206439.62025.97.

6. Viticchi G, Falsetti L, Vernieri F, Altamura C, Bartolini M, Luzzi S, et al. Vascular predictors of cognitive decline in patients with mild cognitive impairment. Neurobiology of aging. 2012;33(6):1127 e19.https://doi: 10.1016/j.neurobiolaging.2011.11.027.

7. Novak V. Cognition and Hemodynamics. Current cardiovascular risk reports. 2012;6(5):380-96. doi: 10.1007/s12170-012-0260-2.

8. Salvadori E, Pasi M, Poggesi A, Chiti G, Inzitari D, Pantoni L. Predictive value of MoCA in the acute phase of stroke on the diagnosis of mid-term cognitive impairment. Journal of neurology. 2013;260(9):2220-7. https://doi: 10.1007/s00415-013-6962-7.

9. Nasreddine ZS, Phillips NA, Bedirian V, Charbonneau S, Whitehead V, Collin I, et al. The Montreal Cognitive Assessment, MoCA: a brief screening tool for mild cognitive impairment. Journal of the American Geriatrics Society. 2005;53(4):695-9.https://doi: 10.1111/j.1532-5415.2005.53221.x.

10. Bugarski Ignjatovic V, Semnic M, Gebauer Bukurov K, Kozic D. Cognitive impairment and functional ability in the acute phase of ischemic stroke. European review for medical and pharmacological sciences. 2015;19(17):3251-6.

11. Webb AJ, Pendlebury ST, Li L, Simoni M, Lovett N, Mehta Z, et al. Validation of the Montreal cognitive assessment versus mini-mental state examination against hypertension and hypertensive arteriopathy after transient ischemic attack or minor stroke. Stroke. 2014;45(11):3337-42. doi: 10.1161/STROKEAHA.114.006309.

12. Cova I, Mele F, Zerini F, Maggiore L, Cucumo V, Brambilla M, et al. Neuropsychological screening in the acute phase of cerebrovascular diseases. Acta neurologica Scandinavica. 2020;142 4:377-84; doi: 10.1111/ane.13319.

13. Lee SH, Park SY, Jang MU, Kim Y, Lee J, Kim C, et al. Association between Osteoporosis and Cognitive Impairment during the Acute and Recovery Phases of Ischemic Stroke. Medicina. 2020;56(6):307. doi: 10.3390/medicina56060307.

14. Biesbroek JM, Weaver NA, Biessels GJ. Lesion location and cognitive impact of cerebral small vessel disease. Clinical science. 2017;131(8):715-28. doi: 10.1042/CS20160452.

15. Zhao L, Biesbroek JM, Shi L, Liu W, Kuijf HJ, Chu WW, et al. Strategic infarct location for post-stroke cognitive impairment: A multivariate lesion-symptom mapping study. Journal of cerebral blood flow and metabolism: official journal of the International Society of Cerebral Blood Flow and Metabolism. 2018;38(8):1299-311. doi: 10.1177/0271678X17728162.

16. Weaver NA, Kuijf HJ, Aben HP, Abrigo J, Bae HJ, Barbay M, et al. Strategic infarct locations for poststroke cognitive impairment: a pooled analysis of individual patient data from 12 acute ischaemic stroke cohorts. The Lancet Neurology. 2021;20(6):448-59. https://doi: 10.1016/S14744422(21)00060-0. 
17. Ni L, Zhang B, Yang D, Qin R, Xu H, Ma J, et al. Lower Cerebrovascular Reactivity Contributed to White Matter Hyperintensity-Related Cognitive Impairment: A Resting-State Functional MRI Study. Journal of magnetic resonance imaging: JMRI. 2021;53(3):703-11. doi: 10.1002/jmri.27376.

18. Hashimoto A, Mikami T, Komatsu K, Noshiro S, Hirano T, Wanibuchi M, et al. Assessment of Hemodynamic Compromise Using Computed Tomography Perfusion in Combination with (123)I-IMP Single-Photon Emission Computed Tomography without Acetazolamide Challenge Test. Journal of stroke and cerebrovascular diseases: the official journal of National Stroke Association. 2017;26(3):627-35. doi: 10.1016/j.jstrokecerebrovasdis.2016.11.013.

19. Chen A, Shyr MH, Chen TY, Lai HY, Lin CC, Yen PS. Dynamic CT perfusion imaging with acetazolamide challenge for evaluation of patients with unilateral cerebrovascular steno-occlusive disease. AJNR American journal of neuroradiology. 2006;27(9):1876-81. PMCID: PMC7977914

20. Ellis MJ, Ryner LN, Sobczyk O, Fierstra J, Mikulis DJ, Fisher JA, et al. Neuroimaging Assessment of Cerebrovascular Reactivity in Concussion: Current Concepts, Methodological Considerations, and Review of the Literature. Frontiers in neurology. 2016;7:61. doi: 10.3389/fneur.2016.00061.

21. Ogasawara K, Ogawa A, Yoshimoto T. Cerebrovascular reactivity to acetazolamide and outcome in patients with symptomatic internal carotid or middle cerebral artery occlusion: a xenon-133 singlephoton emission computed tomography study. Stroke. 2002;33(7):1857-62. doi: 10.1161/01.str.0000019511.81583.a8.

22. Yin X, Zhou Y, Yan S, Lou M. Effects of Cerebral Blood Flow and White Matter Integrity on Cognition in CADASIL Patients. Frontiers in psychiatry. 2018;9:741. doi: 10.3389/fpsyt.2018.00741.

\section{Tables}

Tables 1-4 are available in the Supplementary Files section.

\section{Figures}




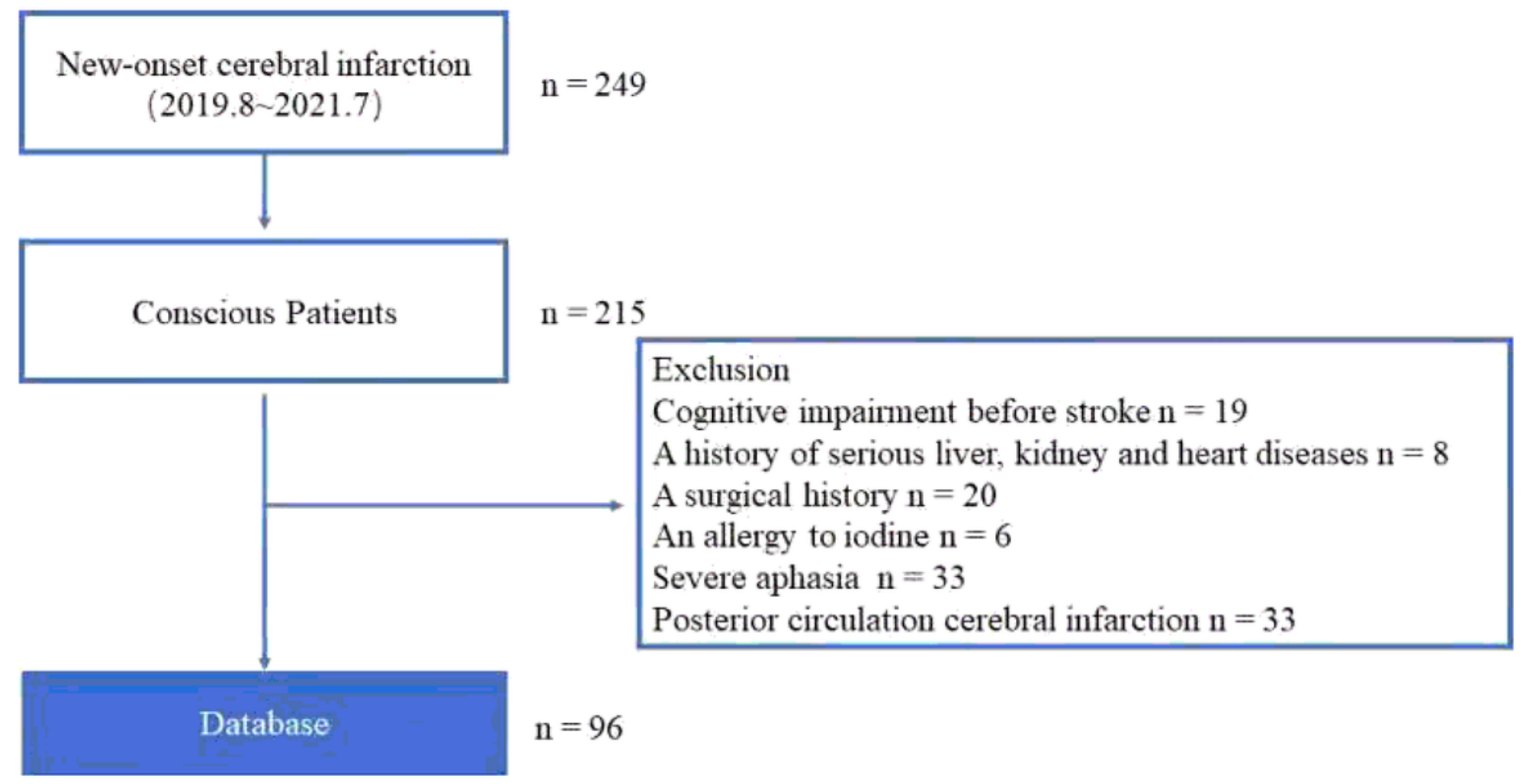

Figure 1

Flow chart of recruitment and exclusion of patients. 
A

$$
\mathrm{P}=0.007
$$

120

100

80

60

40

20

0

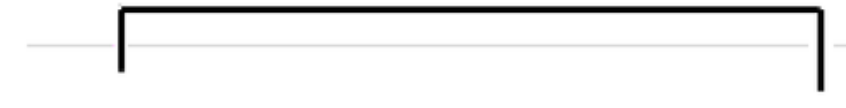

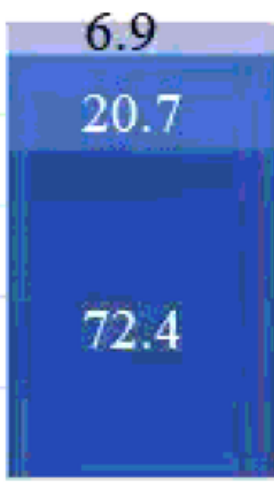

Control

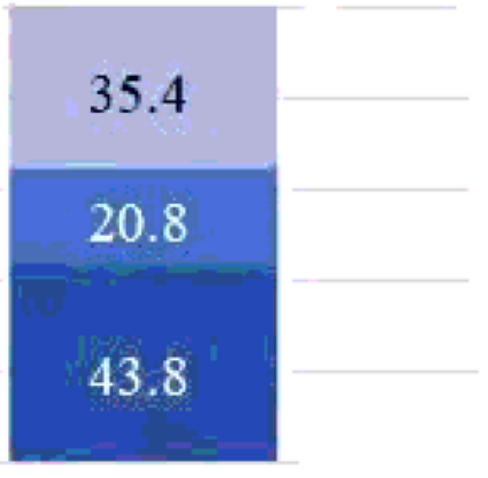

Stroke

B

$$
\mathrm{P}=0.301
$$

120

100

80

60

40

20

0
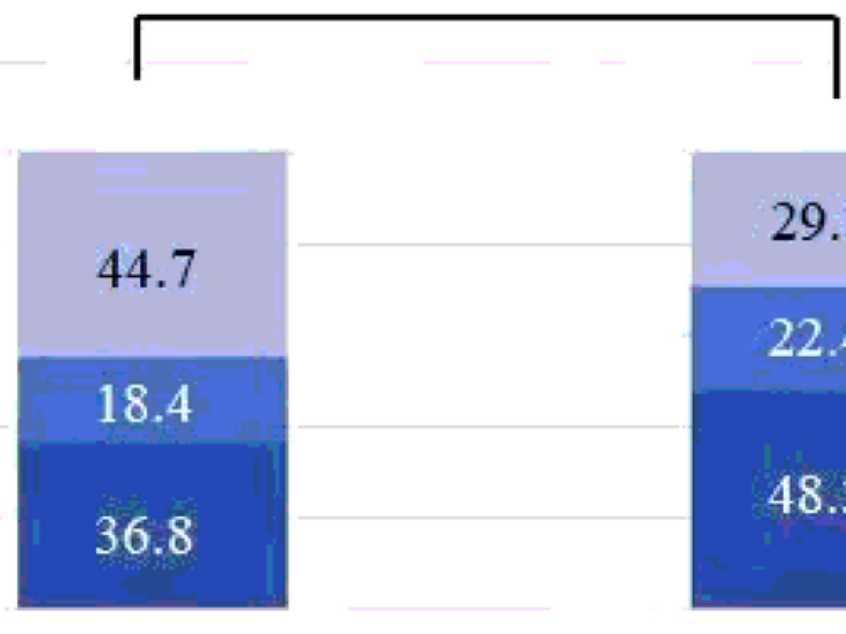

29.3

22.4

48.3

Left infarction

Right infarction

- MoCA 25-30 MoCA 20-24 MoCA 0-19

Figure 2

Cognitive impairment at the acute stroke phase. (A) Cognitive impairment appeared more in patients with new-onset anterior circulation cerebral infarction than in controls ( $56.3 \%$ versus $27.6 \%, p=0.007)$. (B) The cognitive impairment of the left infarction group did not differ from the right infarction group $(p=0.301)$.

\section{Supplementary Files}


This is a list of supplementary files associated with this preprint. Click to download.

- Table1.docx

- Table2.docx

- Table3.docx

- Table4.docx 